\title{
ON THE GAUSS-GREEN THEOREM
}

\author{
B. D. CRAVEN
}

(Received 26 October 1966; revised 25 April 1967)

\section{Introduction}

In a previous paper [1], Green's theorem for line integrals in the plane was proved, for Riemann integration, assuming the integrability of $Q_{x}-P_{y}$, where $P(x, y)$ and $Q(x, y)$ are the functions involved, but not the integrability of the individual partial derivatives $Q_{x}$ and $P_{y}$. In the present paper, this result is extended to a proof of the Gauss-Green theorem for $p$-space $(p \geqq 2)$, for Lebesgue integration, under analogous hypotheses. The theorem is proved in the form

$$
\int_{\Omega} \operatorname{div} g(x) d \mu_{p}(x)=\int_{\partial \Omega} g(x) \cdot v(x) d \Phi(x)
$$

where $\Omega$ is a bounded open set in $R^{p}$ ( $p$-space), with boundary $\partial \Omega$; $g(x)=\left(g\left(x_{1}\right), \cdots, g\left(x_{p}\right)\right)$ is a $p$-vector valued function of $x=\left(x_{1}, \cdots, x_{p}\right)$, continuous in the closure of $\Omega$;

$$
\operatorname{div} g(x)=\sum_{i=1}^{p} \frac{\partial g_{i}(x)}{\partial x_{i}}
$$

$\mu_{\nu}(x)$ is $p$-dimensional Lebesgue measure; $v(x)=\left(v_{1}(x), \cdots, v_{p}(x)\right)$ and $\Phi(x)$ are suitably defined unit exterior normal and surface area on the 'surface' $\partial \Omega$; and $g(x) \cdot v(x)$ denotes inner product of $p$-vectors.

In analogy with the plane case, $\operatorname{div} g(x)$ is assumed finite, except on a suitably restricted 'exceptional set', and Lebesgue integrable on $\Omega$ but the individual partial derivatives $\partial g_{i}(x) / \partial x_{i}$ need not be integrable; and $\partial \Omega$ is assumed to have finite Hausdorff $(p-1)$-measure, and to satisfy a weak continuity condition. The hypothesis on Hausdorff measure, which is analogous to the requirement in [1] that the plane curve is rectifiable, is equivalent to a hypothesis on covering $\partial \Omega$ by cubes, analogous to Potts' Lemma [2] on covering a rectifiable plane curve by squares.

Other authors have assumed that the individual partial derivatives are integrable. Notably, Federer [3], [4], [5] proves the theorem, for suitable scalar $f(x)$, in the form

$$
\int_{\Omega} \frac{\partial f(x)}{\partial x_{i}} d \mu_{p}(x)=\int_{\partial \Omega} f(x) v_{i}(x) d \Phi(x)
$$


and Michael [6] proves (2) with a multiplicity factor inserted. Both assume, however, that $\partial f / \partial x_{i}$ is integrable over $\Omega$.

The proof of (1) depends, not on the detailed definitions of $\nu(x)$ and $\Phi(x)$, but on the following properties assumed for those functions:

(I) $v(x)$ is a Borel-measurable function of $x$, which reduces to the geometric exterior normal to $\Omega$ whenever $\partial \Omega$ is differentiable at $x ; \nu(x)=0$ by convention wherever a normal is undefined.

(II) If $\nu(x)$ and $v^{*}(x)$ denote the unit exterior normals to $\Omega$ and its complement at the point $x \in \partial \Omega$, then $\nu^{*}(x)=-v(x)$.

(III) $\Phi(S)$ is a Carathéodory outer measure ([7] $\S 235$ ) for subsets $S$ of $\partial \Omega$, which equals geometric $(p-1)$-dimensional area in the neighbourhood of any point where the surface $\partial \Omega$ is differentiable. $[\Phi(x)$ denotes $\Phi(S)$ for $S=\left\{y: y_{i} \leqq x_{i}, i=1,2, \cdots, p\right\}$.

(IV) If $\partial \Omega$ denotes the entire boundary of any bounded open set $\Omega$, for which $\Phi(\partial \Omega)<\infty$, then

$$
\int_{\partial \Omega} v_{i}(x) d \Phi(x)=0 \quad(i=1,2, \cdots, p) .
$$

Federer ([3] and [4]) defines a normal $\nu(x)$, which restricts $\Omega$ merely to be a bounded open set, and shows that this $v(x)$, together with $\Phi(S)$ defined as Hausdorff $(p-1)$-measure on $\partial \Omega$, satisfy (I), (II), (III), and (2). It $C$ is any constant vector, then

$$
\begin{aligned}
\int_{\partial \Omega} C \cdot \nu(x) d \Phi(x) & =\sum_{i=1}^{p} C_{i} \int_{\partial \Omega} \nu_{i}(x) d \Phi(x) \\
& =0 \text { from (2), }
\end{aligned}
$$

so that (IV) also holds. It is not obvious whether any other extensions of normal and area exist, satisfying (I) to (IV), but if they do, then Theorems 1, 2, 3 of this paper remain valid for them.

\section{Boundary surface}

If $C$ is a rectifiable plane curve, of length $L$, then Lemma 2 of Potts [2] states that there is a covering $M_{\delta}$ of $L$ by at most $4(L / \delta)+4$ closed squares, each of side $\delta$, with disjoint interiors and sides parallel to the axes. Hence, if $K=8 L$, a constant depending only on $C, M_{\delta}$ consists of at most $K / \delta$ squares of side $\delta$, whose total area $K \delta \rightarrow 0$ as $\delta \rightarrow 0$, and whose total perimeter is less than $4 K$, a bound independent of $\delta$. This fact suggests the following generalization to $R^{p}$. Let 'cube' denote ' $p$-dimensional hypercube with edges parallel to the axes'. A 'surface' $E((p-1)$-dimensional manifold) in $R^{p}$ will be said to satisfy the 'Potts condition' if, for a sequence of values of $\delta \downarrow 0, E$ can be covered by a finite collection $M_{\delta}$ of closed cubes $A_{i}$ with 
disjoint interiors, such that the edge $\delta_{i}$ of $A_{i}$ is less than $\delta$, for each $i$, and $\sum_{i} \delta_{i}^{p-1}<K$, a constant independent of $\delta$. Denote by $M_{\delta}^{*}$ the union of the cubes of $M_{\delta}$. It follows that the total $p$-dimensional volume of $M_{\delta}^{*}$ is less than $K \delta$, so $\rightarrow 0$ with $\delta$, and the total $(p-1)$-dimensional surface area of the cubes of $M_{\delta}$ is less than $2 p K$, for all $\delta$. The 'Potts condition' is further characterized by the following two Lemmas.

Lemma 1. The boundary $E$ of a bounded open set in $R^{p}$ satisfies the Potts condition if and only if its Hausdorff $(p-1)$-measure, $\Phi(E)$, is finite.

Proof. Hausdorff measure is defined [5] as

$$
\begin{aligned}
\Phi(E)=2^{-p+1} \alpha_{p-1} \lim _{r \rightarrow 0+} & {\left[\operatorname { i n f } \left\{\sum_{j=1}^{\infty}\left(\operatorname{diam} B_{j}\right)^{p-1}:\right.\right.} \\
& \left.\left.E \subset \bigcup_{j=1}^{\infty} B_{j} ; \operatorname{diam} B_{j}<r, j=1,2, \cdots\right\}\right]
\end{aligned}
$$

where $\alpha_{p-1}=$ volume of $(p-1)$-dimensional unit sphere. Let $E$ satisfy the Potts condition. For any $r>0$, there is a covering $M_{\delta}$ of $E$ by cubes $A_{i}$ of edge $<\delta$, and therefore of diameter $<\delta p^{\frac{1}{2}}<r$, by choice of $\delta$, such that

$$
\sum_{i}\left(\operatorname{diam} A_{i}\right)^{p-1}=\left(p^{\frac{1}{2}}\right)^{p-1} \sum_{i} \delta_{i}^{p-1}<K\left(p^{\frac{1}{2}}\right)^{p-1},
$$

a constant independent of $r$, consequently, from $(5), \Phi(E)<\infty$.

The converse is Theorem 4.1 of Michael [8], noting that $E$ is compact.

Lemma 2. Let $C$ be a plane closed Jordan curve. Then $C$ satisfies the Potts condition if and only if $C$ is rectifiable.

Proof. If $C$ is rectifiable, then $C$ satisfies the Potts condition, by Potts' Lemma. Conversely, let $C$ satisfy the Potts condition. Then $C$ is bounded. Choose any $n$ distinct points $P_{0}, P_{1}, \cdots, P_{n-1}$ on $C$, taken in order around $C$; denote $P_{n}=P_{0}$. Cover each $P_{i}$ by a square $K_{i}$, whose edge $<1 / n$. Let $C_{i}$ denote that part of the $\operatorname{arc} P_{i-1} P_{i}$ which lies outside Int $\left(K_{i-1} \cup K_{i}\right)$. Since the $C_{i}$ are disjoint compact, there is a Potts covering $M$ of $C$, such that each $C_{i}$ is covered by a union $M_{i}$ of squares of $M$, and the $M_{i}$ are disjoint. There are points $Q_{i-1}^{\prime} \in K_{i-1} \cap \partial M_{i}$ and $Q_{i}^{\prime \prime} \in K_{i} \cap \partial M_{i}$, where $\partial M_{i}$ denotes the boundary of $M_{i}$. There is an arc, of length $b_{i}$ say, joining $Q_{i-1}^{\prime}$ to $Q_{i}^{\prime \prime}$, consisting of parts of edges of squares of $M_{i}$. Then, if $d$ denotes distance, and $K$ is the constant of the Potts hypothesis,

$$
\begin{aligned}
\sum_{i=1}^{n} d\left(P_{i-1}, P_{i}\right) & \leqq \sum_{i=1}^{n}\left\{d\left(P_{i-1}, Q_{r}^{\prime}\right)+b_{i}+d\left(Q_{i}^{\prime \prime}, P_{i}\right)\right\} \\
& \leqq n \cdot \frac{\sqrt{ } 2}{n}+4 K+\frac{n \sqrt{ } 2}{n}=2 \sqrt{ } 2+4 K,
\end{aligned}
$$

a bound independent of the $P_{i}$. So $C$ is rectifiable. 


\section{Admissible domains}

Let $\Omega$ be a bounded open subset of $R^{p}$, whose boundary $\partial \Omega$ is a countable union of disjoint continuous images $E_{k}$ of $S^{p-1}$, the $(p-1)$ dimensional unit sphere. Let $V=\cup V_{k}$, where the $V_{k}$ are countably many disjoint copies of $S^{p-1}$ in $R^{p}$. Now $E_{k}=f_{k}\left(V_{k}\right)$, where each $f_{k}$ is continuous, so that $\partial \Omega=f(V)$, where $f \mid V_{k}=f_{k}$, and $f$ is continuous. (The set $V$ may be taken instead as a countable union of disjoint closed intervals in $R$.)

If $\partial \Omega$ is topologised as a subspace of $R^{p}$, then the sets

$$
A=\partial \Omega \cap\left\{x: x_{i}<\alpha\right\} \text { and } B=\partial \Omega \cap\left\{x: x_{i}>\alpha\right\}
$$

are open in $\partial \Omega$, so their inverse images $f^{-1} A$ and $f^{-1} B$ are open in $V$, and therefore consist of at most countably many disjoint arcwise-connected components. Consequently, if $K$ is any open cube in $R^{p}, \partial \Omega \cap K$ consists of at most countably many components.

Let $L_{i}(\alpha)=\partial \Omega \cap\left\{x: x_{i} \leqq \alpha\right\}$. Since $\Phi$ is monotone, $\Psi_{1}\left(\alpha_{1}\right)=\Phi\left(L_{1}\left(\alpha_{1}\right)\right)$ is a nondecreasing function of $\alpha_{1}$, so there is a countable dense set $D_{1}$ of $\alpha_{1}$ on which $\Psi_{1}$ is continuous. Likewise, for each $\alpha_{1} \in D_{1}$,

$$
\Psi_{2}\left(\alpha_{1}, \alpha_{2}\right)=\Phi\left(L_{1}\left(\alpha_{1}\right) \cap L_{2}\left(\alpha_{2}\right)\right)
$$

is a nondecreasing function of $\alpha_{2}$, so there is a countable dense set $D_{2}$ of $\alpha_{2}$ such that $\Psi_{2}$ is continuous for $\alpha_{1} \in D_{1}, \alpha_{2} \in D_{2}$; and so on. The planes $x_{i}=\alpha_{i} \in D_{i}(i=1,2, \cdots, p)$ will be called admissible planes. Since they form a dense family, the cubes used in Potts coverings can be replaced by cuboids bounded by admissible planes, with arbitrarily little change in the bounds previously obtained; this will be assumed henceforth. If $W$ is any open cuboid bounded by admissible planes, then any component of $W \cap \partial \Omega$ will be called an admissible domain in $\partial \Omega$. then

Lemma 3. If $A_{i}(i=1,2, \cdots)$ are disjoint admissible domains in $\partial \Omega$,

(i) $\Phi\left(\overline{A_{i}}\right)=\Phi\left(A_{i}\right)$, where $\overline{A_{i}}=$ closure of $A_{i}$ in $\partial \Omega$;

(ii) $\Phi\left(A_{1}+A_{2}\right)=\Phi\left(A_{1}\right)+\Phi\left(A_{2}\right)$, where $A_{1}+A_{2}$ now denotes the interior of $\overline{A_{1}} \cup \overline{A_{2}}$; denote also $A_{1}+A_{2}+\cdots+A_{n}+\cdots=$ Interior of $\bigcup_{1}^{\infty} \overline{A_{i}}$;

(iii) if $A_{0}=A_{1}+A_{2}+\cdots+A_{n}+\cdots$ is also admissible, then

$$
\Phi\left(A_{0}\right)=\sum_{1}^{\infty} \Phi\left(A_{n}\right)
$$

(iv) $A_{i}$ is $\Phi$-measurable;

(v) if $f(x)$ is bounded Borel-measurable, then

$$
\int_{A_{1}+A_{2}} f d \Phi=\int_{A_{1}} f d \Phi+\int_{A_{2}} f d \Phi .
$$


Proof. (i) If $W$ is an open cuboid bounded by admissible planes, then the continuity of $\Phi$ on admissible planes implies that there is a larger cuboid $W_{\varepsilon}$, obtained by displacing outward each boundary plane of $W$, such that $\bar{W} \subset W_{\varepsilon}$, and $\Phi\left(W_{\varepsilon} \cap \partial \Omega\right)<\Phi(W \cap \partial \Omega)+\varepsilon$. So if $A$ is an admissible domain, there is an admissible domain $A_{\varepsilon} \supset \bar{A}$ with $\Phi\left(A_{\varepsilon}\right)<\Phi(A)+\varepsilon$; which implies (i).

(ii) Define distance $d$ on $\partial \Omega$ as the restriction to $\partial \Omega$ of distance in $R^{p}$. Since $A_{1} \cap A_{2}=\emptyset, C=\overline{A_{1}} \cap \overline{A_{2}}$ is contained in the frontiers (in $\partial \Omega$ ) of $A_{1}$ and $A_{2}$. By the definition of admissible domain, these frontier points are boundary points of finitely many cuboids bounded by admissible planes. These planes may be covered by a finite union $G$ of open cuboids, such that $\Phi(D)<\varepsilon$, where $C \subset D=G \cap \partial \Omega$. Then the sets $\overline{A_{i}}-D=A_{i}-D(i=1,2)$ are disjoint closed sets in $\partial \Omega$; therefore $d\left(A_{1}-D, A_{2}-D\right)>0$. Since $\Phi$ is a Carathéodory outer measure, it is additive on $A_{1}-D$ and $A_{2}-D$, and the result follows.

(iii) Since $A_{1}+\cdots+A_{n} \subset \overline{A_{0}}$,

$$
\sum_{1}^{n} \Phi\left(A_{i}\right) \leqq \Phi\left(\overline{A_{0}}\right)=\Phi\left(A_{0}\right) \quad \text { by (ii) and (i); }
$$

since $\Phi$ is subadditive,

$$
\Phi\left(\overline{A_{0}}\right) \leqq \sum_{1}^{\infty} \Phi\left(\overline{A_{i}}\right)=\sum_{1}^{\infty} \Phi\left(A_{i}\right) \quad \text { by (i). }
$$

(iv) Since $A_{i}$ is open in $\partial \Omega$, and $\Phi$ is a Carathéodory outer measure on $\partial \Omega, A_{i}$ is measurable (Carathéodory [7], $\S 238$ and $\S 251$ ).

(v) From (ii) and (iv), it readily follows that, for any Borel set $B$ (i.e. any set obtained from admissible domains by countably many unions and intersections) $\Phi\left(B \cap\left(A_{1}+A_{2}\right)\right)=\Phi\left(B \cap A_{1}\right)+\left(B \cap A_{2}\right)$; and this leads readily to $(\mathrm{v})$.

LemMa 4. If $f(x)$ is bounded Borel-measurable; $A_{1}, A_{2}, \cdots$ are disjoint admissible domains; and $A=A_{1}+A_{2}+\cdots$ is an admissible domain, with $\Phi(A)<\infty$; then, independently of the order of summation,

$$
\int_{A} f d \Phi=\sum_{i=1}^{\infty} \int_{A_{i}} f d \Phi
$$

Proof. Since $\Phi(A)<\infty, \int_{A}$ is finite, and by Lemma 3 (iii), so is each $\int_{A_{i}}$. Suppose that some sequence of partial sums of the series (6), summed in some order, converges to a limit $\lambda$, where $\left|\lambda-\int_{A}\right|=3 \delta>0$. Then

$$
\left|\sum_{i \in N_{r}} \int_{A_{i}}-\lambda\right|<\delta
$$


for an expanding sequence of finite sets $N_{r} \uparrow N$, the set of all positive integers. If $F_{r}=A_{1}+\cdots+A_{r}$ and $G_{r}=A-A_{r}$, then by Lemma 3 (iii)

$$
\Phi\left(G_{r}\right) \leqq \sum_{N-N_{r}} \Phi\left(\overline{A_{r}}\right)=\sum_{N-N_{r}} \Phi\left(A_{i}\right)<\delta / \sup |f|
$$

by choice of $r$, since $\sum \Phi\left(A_{i}\right)<\infty$. Since $F_{r}, G_{r}$ are disjoint measurable sets, $3 \delta=\left|\int_{F_{r}}+\int_{G_{r}}-\lambda\right|=\left|\sum_{N_{r}} \int_{A_{i}}+\int_{G_{r}}-\lambda\right| \leqq\left|\sum_{N_{r}} \int_{A_{i}}-\lambda\right|+\sup |f| \Phi\left(G_{r}\right) \leqq \delta+\delta$, so that $\delta=0$.

\section{Gauss-Green theorem}

TheOREM 1. Let $\Omega$ be a bounded open subset of $R^{p}$, whose boundary $\partial \Omega$ (i) satisfies the Potts condition, and (ii) is a countable union of disjoint continuous images of $S^{p-1}$. Let $g: \bar{\Omega} \rightarrow R^{p}$ be continuous on $\bar{\Omega}$. Let div $g$ be Lebesgue-integrable on $\Omega$. For every cuboid $\Gamma \subset \Omega$, let the Gauss-Green theorem (1) hold, with $\Omega, \partial \Omega$ replaced by $\Gamma, \partial \Omega$. Then (1) holds for $\Omega, \partial \Omega$.

Proof. Let $M_{\delta}$ be a Potts covering of $\partial \Omega$, consisting of closed cuboids $A_{i}$. Denote the interior of $A_{i}$ by $A_{i}^{0}$. Let $C_{\delta}$ denote the union of those relatively open subsets of the boundary planes of the $A_{i}$ which lie in $M_{\delta}^{*} \cap \Omega$. Then, by definition of Potts covering, $\mu_{p}\left(M_{\delta}^{*}\right)<K \delta$ and $\mu_{p-1}\left(C_{\delta}\right)<2 p K$. Let $h(x)=\operatorname{div} g(x)$ for $x \in \Omega, h(x)=0$ for $x \notin \Omega$. Then

Since $h \in L\left(R^{p}\right)$,

$$
\int_{\Omega} \operatorname{div} g d \mu_{p}=\int_{R^{p}} h d \mu_{p} .
$$

$$
\left|\int_{M g} h d \mu_{p}\right|<\varepsilon
$$

if $\mu_{p}\left(M_{\delta}^{*}\right)<\Delta(\varepsilon)$. So, if $W=\Omega-M_{\delta}^{*}$ and $\delta<K^{-1} \Delta(\varepsilon)$,

$$
\left|\int_{\Omega} \operatorname{div} g d \mu_{p}-\int_{W} h d \mu_{p}\right|<\varepsilon
$$

The set $A_{i} \cap \Omega$ has boundary $\rho_{i}=\alpha_{i} \cup \sigma_{i} \cup \lambda_{i}$, where $\alpha_{i}=A_{i}^{0} \cap \partial \Omega$ is the union of (at most) countably many admissible domains $\alpha_{i j}$, the relatively open set $\sigma_{i}=\partial A_{i} \cap \Omega$ is the union of (at most) countably many components $\beta_{i j}$ of $C_{\delta}-\partial W$ and $\gamma_{i j}$ of $\partial W$, and $\lambda_{i}=\partial A_{i} \cap \partial \Omega$ satisfies $\Phi\left(\lambda_{i}\right)=0$, since $A_{i}$ is bounded by admissible planes. The frontiers of the open sets $\beta_{i j}$ and $\gamma_{i j}$, in the relative topology of $\partial A_{i}$, are contained in $\lambda_{i}$. Consequently, the results of Lemmas 3 and 4 apply also to the $\beta_{i j}$ and $\gamma_{i j}$; these sets will also be called 'admissible domains'.

In terms of the set composition + of Lemma $3, \rho_{i}$ is the sum, over countably many indices $j$, of the $\alpha_{i j}, \beta_{i j}, \gamma_{i j}$. The proof of Theorem 1 consists 
essentially in recombining the corresponding integrals in a different order; this process is validated by Lemma 4 , which also shows that the frontier points (in the relative topology) of the admissible domains make no contribution.

Attach to each point $x \in \rho_{i}$ the unit exterior normal $\nu(x)$. For $x \in \beta_{i j}$, two normals are possible, oppositely directed, depending on which $\rho_{i}$ is chosen; in the following summation, each $\beta_{i j}$ contributes twice, once for each normal. With integrand $g \cdot v d \Phi$,

$$
\begin{array}{rlrl}
\int_{\partial \Omega} & =\sum_{i, j} \int_{\alpha_{i j}} & & \text { by Lemma 4 } \\
& =\sum_{i, j} \int_{\alpha_{i j}}+\sum_{i, j} \int_{\beta_{i j}}+\sum_{i, j} \int_{\gamma_{i j}}-\sum_{i, j} \gamma_{i j} \text { since } \sum_{i, j} \int_{\beta_{i j}}=0 & \\
& =\sum_{i} \sum_{j}\left(\int_{\alpha_{i j}}+\int_{\beta_{i j}}+\int_{\gamma_{i j}}\right)-\sum_{i, j} \int_{\gamma_{i j}} & & \text { by Lemma 4 } \\
& =\sum_{i, j} \int_{\rho_{i j}}+\int_{\partial W} & & \text { by Lemma 4. }
\end{array}
$$

Since $g$ is continuous on the compact set $\bar{\Omega}$, and $\mu_{p}\left(M_{\delta}\right) \rightarrow 0$ as $\delta \rightarrow 0$, there is $\delta$ such that the oscillation of $g(x)$ in the closure of each $A_{i} \cap \Omega$ is less than $\varepsilon$. So, for $\delta$ sufficiently small, there corresponds to each $\rho_{i}$ a constant vector $c_{i}$ such that, for $x \in \rho_{i}$,

Hence

$$
g(x)=c_{i}+\eta_{i}(x) \text { where }\left|\eta_{i}(x)\right|<\varepsilon .
$$

so that

$$
\begin{aligned}
\int_{\rho_{i j}} g \cdot v d \Phi & =\int_{\rho_{i j}} c_{i} \cdot v d \Phi+\int_{\rho_{i j}} \eta_{i} \cdot v d \Phi \\
& =\int_{\rho_{i j}} \eta_{i} \cdot v d \Phi
\end{aligned}
$$

$$
\begin{aligned}
\left|\sum_{i j} \int_{\rho_{i j}} g \cdot v d \Phi\right| & \leqq \varepsilon \sum_{i j} \Phi\left(\rho_{i j}\right) \\
& \leqq \varepsilon\left(2 \sum_{i} \Phi\left(\partial A_{i}\right)+\Phi(\partial \Omega)\right) \quad \text { by Lemma } 3 \text { (iii) } \\
& \leqq \varepsilon(4 p K+\Phi(\partial \Omega))
\end{aligned}
$$

where $K$ is the constant of the family of Potts coverings.

Now the Gauss-Green theorem applies, by hypothesis, to $W$, which is a finite union of cuboids $C \Omega$. Combining this with (7) and (9),

$$
\left|\int_{\Omega} \operatorname{div} g d \mu_{p}-\int_{\partial \Omega} g \cdot v d \Phi\right| \leqq B \cdot \varepsilon
$$

for constant $B$; which proves the theorem. 
Lemma 5. (Saks [9], page 198.) Let w be a real function of one variable, such that $w^{\prime}(x)$ exists p.p. in $[a, b]$; let $F$ be a closed non-empty subset of $[a, b]$; let $N$ be a finite constant such that

Then

$$
\left|w\left(x_{2}\right)-w\left(x_{1}\right)\right| \leqq N\left|x_{2}-x_{1}\right| \text { whenever } x_{1} \in F \text { and } x_{2} \in[a, b] .
$$

$$
\left|w(b)-w(a)-\int_{F} w^{\prime}(x) d x\right| \leqq N\left(b-a-\mu_{1}(F)\right) .
$$

Proof. (Saks) Let $u(x)=w(x)$ on $F \cup\{a, b\}$, and linear on the complementary intervals. Then $u(x)$ is Lipschitz, therefore absolutely continuous. Hence

$$
w(b)-w(a)=u(b)-u(a)=\int_{a}^{b} u^{\prime}(x) d x .
$$

But $u^{\prime}(x)=w^{\prime}(x)$ p.p. in $F$, and $\left|u^{\prime}(x)\right| \leqq N$ at each $x \epsilon^{\prime} F$, which proves the result.

THEOREM 2. Let $W$ be an open cuboid in $R^{p}$; let $K$ be an open cuboid containing $\bar{W}$. Let $g(x)$ be continuous on $K$; let div $g(x)$ be finite for all $x \in K$ and Lebesgue integrable on $W$. Then the Gauss-Green theorem (1) holds for $W$, $\partial W$.

Proof. A point $x \in \bar{W}$ will be called admissible if it has an open neighbourhood $N(x) \subset K$, such that for every cuboid $C \subset N(x)$, (1) holds for $C, \partial C$. Let $F$ denote the complement, with respect to $\bar{W}$, of the set of admissible points. From its construction, $F$ is closed. Suppose that $F$ is not empty; this will lead to a contradiction.

For $n=1,2, \cdots$, denote by $F_{n}$ the set of points $x$ for which

$$
\begin{aligned}
\max _{i=1,2, \cdots, p} & \mid g\left(x_{1}, \cdots, x_{i-1}, x_{i}+h, x_{i+1}, \cdots, x_{p}\right) \\
& -g\left(x_{1}, \cdots, x_{i-1}, x_{i}, x_{i+1}, \cdots, x_{p}\right)|\leqq n| h \mid \text { for }|h|<n^{-1} .
\end{aligned}
$$

Since $\partial g_{i}(x) / \partial x_{i}$ is finite for all $x, \bar{W} \subset \cup_{n} F_{n}$. Then, according to Baire's category theorem ([9] page 55) there is an open cuboid $I$ such that $F \cap F_{N}$ is dense in $F \cap I$ for some integer $N$. Since also $F$ and $F_{N}$ are closed, $\emptyset \neq I \cap F \subset I \cap\left(F \cap F_{N}\right) \subset F_{N}$. Let $x_{0} \in I \cap F$. Let $Q$ be any closed cuboid of diameter $\leqq N^{-1}$, where $x_{0} \in Q \subset I$.

Given $\delta>0$, there is a countable covering of $E=F \cap Q$ by open cuboids $G$, such that

$$
\sum_{1}^{\infty} \mu_{p}\left(G_{j}\right)<\mu_{p}(F \cap Q)+\delta
$$

Since $F \cap Q$ is compact, a finite subset of the $G_{j}$ covers $F \cap Q$. Since also $\mu_{p}\left(\bar{G}_{j}\right)=\mu_{p}\left(G_{j}\right)$, there is a finite covering of $F \cap Q$ by closed cuboids $S_{j}$ 
$(j=1, \cdots, r)$ which may be assumed to have disjoint interiors, and to lie within $Q$, such that

$$
\sum_{1}^{r} \mu_{p}\left(S_{j}\right)<\mu_{p}(F \cap Q)+\delta .
$$

Let $S_{j}$ be the cuboid $a_{j} \leqq x_{j} \leqq b_{j}(j=1, \cdots, p)$. Let the line specified by fixed values of $x_{1}, \cdots, x_{i-1}, x_{i+1}, \cdots, x_{p}$ intersect $F \cap S_{j}$ in the set $T_{i}=T_{i}\left(x_{1}, \cdots, x_{i-1}, x_{i+1}, \cdots, x_{p}\right)$, whose linear measure is $\mu_{1}\left(T_{i}\right)$. Then, from Lemma 5 ,

$$
\begin{aligned}
& \psi\left(x_{1}, \cdots, x_{i-1} ; x_{i+1}, \cdots, x_{p}\right) \\
& \equiv \mid g_{i}\left(x_{1}, \cdots, x_{i-1}, b_{i}, x_{i+1}, \cdots, x_{p}\right)-g_{i}\left(x_{1}, \cdots, x_{i-1}, a_{i}, x_{i+1}, \cdots, x_{p}\right) \\
&\left.\quad-\int_{T_{i}} \frac{\partial g_{i}}{\partial x_{i}}\left(x_{1}, \cdots, x_{i-1}, x_{i}, x_{i+1}, \cdots, x_{p}\right) d x_{i}\right) \mid \\
& \leqq N \cdot\left(b_{i}-a_{i}-\mu_{1}\left(T_{i}\right)\right) .
\end{aligned}
$$

So, integrating with respect to $x_{1}, \cdots, x_{i-1}, x_{i+1}, \cdots, x_{p}$ over $a_{j} \leqq x_{j} \leqq b_{j}$,

$$
\begin{aligned}
& \left|\int_{\partial S_{j}} g_{i}(x) v_{i}(x) d \Phi(x)-\int_{S_{j} \cap F} \frac{\partial g_{i}(x)}{\partial x_{i}} d \mu_{p}(x)\right| \\
& =\int \psi\left(x_{1}, \cdots, x_{i-1} ; x_{i+1}, \cdots, x_{p}\right) d x_{1} \cdots d x_{i-1} d x_{i+1} \cdots d x_{p}, \\
& \quad \leqq N \int\left(b_{i}-a_{i}-\mu_{1}\left(T_{i}\right)\right) d x_{1} \cdots d x_{i-1} d x_{i+1} \cdots d x_{p} \\
& =N\left(\mu_{p}\left(S_{j}\right)-\mu_{p}\left(S_{j} \cap F\right)\right) \quad \text { by Fubini's theorem. }
\end{aligned}
$$

Define the set function $H(S)$ on closed cuboids $S$ by

$$
p H(S)=\int_{\partial S} g(x) \cdot v(x) d \Phi(x)-\int_{S} \operatorname{div} g(x) d \mu_{p}(x) .
$$

Then $H(S)$ is additive on cuboids whose interiors are disjoint, and, from the definition of $F$,

$$
H(S)=0 \text { if } F \cap S=\emptyset .
$$

Now since $H$ is additive,

$$
\begin{aligned}
\left|H\left(\bigcup_{\mathbf{1}}^{r} S_{j}\right)\right| & \leqq \sum_{1}^{r}\left|H\left(S_{j}\right)\right| \\
& \leqq N \sum_{j=1}^{r}\left[\mu_{p}\left(S_{j}\right)-\mu_{p}\left(S_{j} \cap F\right)+\int_{S_{,}-F}|\operatorname{div} g(x)| d \mu_{p}(x)\right] \text { by (13) } \\
& \leqq N \mu_{p}\left(\left(\bigcup_{1}^{r} S_{j}\right)-F \cap Q\right)+N \int_{\left(\cup S_{j}\right)-(F \cap Q)}|\operatorname{div} g(x)| d \mu_{p}(x) .
\end{aligned}
$$


Since $g$ is integrable over $W$, the integral in (16) can be made less than $\varepsilon /(2 N)$ by choosing $\mu_{p}\left(\left(\cup S_{j}\right)-(F \cap Q)\right)<\Delta(\varepsilon / 2 N)$, say. From (12)

$$
\mu_{p}\left(\left(\cup S_{j}\right)-(F \cap Q)<\min (\varepsilon / 2 N, \Delta(\varepsilon / 2 N))\right.
$$

if $\delta$ is chosen less than the quantity on the right of (17). Hence $\left|H\left(\cup S_{j}\right)\right|<\varepsilon$. Now

$$
\begin{aligned}
|H(Q)| & =\left|H\left(Q-\cup S_{j}\right)+H\left(\cup S_{j}\right)\right| \\
& \leqq\left|H\left(Q-\cup S_{j}\right)\right|+\left|H\left(\cup S_{j}\right)\right| \\
& <\mathbf{0}+\varepsilon,
\end{aligned}
$$

since $Q-S_{j} \subset Q-F$. Since $\varepsilon$ is arbitrary, $H(Q)=0$. Since this is true for every sufficiently small cuboid $Q$ containing $x_{0}$, the assumption $x_{0} \in F$ is contradicted. Hence $F$ is empty.

THeOREM 3. Let $\Omega$ be a bounded open subset of $R^{p}$, whose boundary $\partial \Omega$ satisfies the Potts condition (or equivalently, by Lemma 1, has $\Phi(\partial \Omega)<\infty$ ), and is a countable union of disjoint continuous images of $S^{p-1}$. Let $E$ be a subset of $\Omega$ which satisfies the same hypotheses as $\partial \Omega$. Let the function $g: \bar{\Omega} \rightarrow R^{p}$ be continuous; let div $g$ exist (with finite value) at all points of $\Omega-E$, and be integrable on $\Omega$. Then the Gauss-Green theorem (1) holds for $\Omega, \partial \Omega$.

REMARKs. The topological hypothesis on $\partial \Omega$ is an analog of the hypothesis, in Green's theorem for two dimensions, that the boundary is a closed Jordan curve.

The subset $E$ may consist, e.g., of countably many points, or lines, etc., within $\Omega$, on which one or more derivatives $\partial g_{i} / \partial x_{i}$ fail to exist; since $\mu_{p}(E)=\mathbf{0}$ (from the Potts condition), $\operatorname{div} g$ is defined a.e. on $\Omega$.

The Looman-Menchoff theorem (Saks [9]) states that if $f(z)=u+i v$ is a continuous function of complex $z$ on domain $\Omega$, and $u$ and $v$ have their first partial derivatives finite in $\Omega$ except on a countable set $E$, and satisfy the Cauchy-Riemann equations a.e. in $\Omega$, then $\oint_{C} f(z) d z=0$ for each closed rectangle $C$ in $\Omega$. Theorem 3 of this paper shows that this exceptional set $E$ can be considerably enlarged.

Proof. Let $M$ be a closed Potts covering of $E$, with parameter $\delta$. The hypotheses of Theorem 2, and consequently the Gauss-Green theorem, hold for each cuboid $K \subset \Omega-M$. Therefore, by Theorem 1, the Gauss-Green theorem holds also for $\Omega-M$ and its boundary.

Since $E$ satisfies the same hypotheses as $\partial \Omega$, the arguments which lead to (7) and (9) in the proof of Theorem 1 show also that, for sufficiently small $\delta$, 


$$
\begin{aligned}
& \left|\int_{\Omega} \operatorname{div} g d \mu_{p}-\int_{\Omega-M} \operatorname{div} g d \mu_{p}\right|<\varepsilon \\
& \left|\int_{\partial \Omega}-\int_{\partial(\Omega-M)} g \cdot \nu d \Phi\right|<k \cdot \varepsilon
\end{aligned}
$$

where $k$ is constant. Since $\varepsilon$ is arbitrary, these results combine to prove the Gauss-Green theorem for $\Omega, \partial \Omega$.

\section{Examples}

(I) Theorem 3, or even the two-dimensional Riemann-integral version in [1], is a non-trivial extension of the usual Gauss-Green theorem. An example in two dimensions is as follows.

Let $\Omega$ denote the interior of the unit circle $x_{1}^{2}+x_{2}^{2}=1$. Let

$$
\begin{aligned}
& g_{1}\left(x_{1}, x_{2}\right)=x_{2} r^{2} \sin \pi / r^{4} \\
& g_{2}\left(x_{1}, x_{2}\right)=-x_{1} r^{2} \sin \pi / r^{4}
\end{aligned}
$$

where $r^{2}=x_{1}^{2}+x_{2}^{2}$. Then $g_{1}$ and $g_{2}$ are continuous, and even differentiable, at all points in $\Omega$, since for $r \neq 0$,

$$
\frac{\partial g_{1}}{\partial x_{1}}=-2 x_{1} x_{2} \sin \frac{\pi}{r^{4}}+\frac{4 \pi x_{1} x_{2}}{r^{4}} \cos \frac{\pi}{r^{4}}=-\frac{\partial g_{2}}{\partial x_{2}},
$$

and $\left|\left[g_{1}\left(x_{1}, x_{2}\right)-g_{1}(0,0)\right] / r\right|<r$ (and similarly for $\left.g_{2}\right)$.

Thus $\operatorname{div} g(x)=0$ in $\Omega$, so is integrable, and Green's theorem holds for these functions. But if $\partial g_{1} / \partial x_{1}$ were integrable on $\Omega$, it would follow (since $2 x_{1} x_{2} \sin \pi / r^{4}$ is continuous) that

$$
\iint\left|\frac{x_{1} x_{2}}{r^{4}} \cos \frac{\pi}{r^{4}}\right| d x_{1} d x_{2}<\infty,
$$

hence in polar coordinates,

or (with $r=\dot{S-1}$ )

$$
\int_{0}^{1}\left|\cos \frac{\pi}{r^{4}}\right| \frac{d r}{r}<\infty
$$

$$
\int_{0}^{1}|\cos \pi S| \frac{d S}{S}<\infty
$$

Since this integral diverges, $\partial g_{1} / \partial x_{1}$ is not integrable on $\Omega$, consequently the usual forms of Green's theorem do not apply.

(II) Theorem 3 is untrue if the exceptional set $E$, on which div $g$ fails to exist, is increased to an arbitrary null set (i.e. $\mu_{p}(E)=0$ ). A counterexample for $p=2$ is given by $\Omega=$ unit square $\left(0 \leqq x_{1} \leqq 1,0 \leqq x_{2} \leqq 1\right)$, $g_{2}(x)=0, g_{1}(x)=\phi\left(x_{1}\right) \phi\left(x_{2}\right)$, where $\phi(x)$ is Cantor's monotonic function 
for which $\phi^{\prime}(x)=0$ except on a null set $N$, but $\phi(1)-\phi(0)=1$. Then $\operatorname{div} g=0$ except on the null set $E=N \times N$, so that

$$
\int_{\Omega} \operatorname{div} g d \mu_{2}=0, \text { but } \int_{\partial \Omega} g \cdot \nu d \Phi \neq 0
$$

\section{Acknowledgement}

I am much indebted to Dr. J. H. Michael for helpful discussion on the subject of this paper, and for valuable suggestions concerning the method of proof of Theorem 2. I am also indebted to the referee for pointing out a number of details which required amendment.

\section{References}

[1] B. D. Craven, 'A note on Green's theorem', J. A ustral. Math. Soc. 4 (1964), 289-292.

[2] D. H. Potts, 'A note on Green's theorem', J. Lond. Math. Soc. 26 (1951), 302-304.

[3] W. Federer, 'The Gauss-Green theorem', Trans. Amer. Math. Soc. 58 (1945), 44-76.

[4] W. Federer, 'Coincidence functions and their integrals', Trans. Amer. Math. Soc. 59 (1946), $441-466$.

[5] W. Federer, 'Measure and area,' Bull. Amev. Math. Soc. 58 (1952), 306-378.

[6] J. H. Michael, 'Integration over parametric surfaces', Proc. Lond. Math. Soc., Third Ser. 7 (1957), 616-640.

[7] C. Carathéodory, Vorlesungen über Reelle Funktionen (Teubner, 1927).

[8] J. H. Michael, 'An n-dimensional analogue of Cauchy's integral theorem', J. Austral. Math. Soc. 1 (1960), $171-202$.

[9] S. Saks, Theory of the integral (Second edition).

University of Melbourne. 